\title{
An Unusual Case Of Segmental Clavicle Fracture
}

\author{
R Heywood, J Clasper
}

\begin{abstract}
Fracture of the clavicle is a common traumatic injury and comprises $4 \%$ of all fractures in adults. Amongst these, midshaft injuries account for the majority and medial fractures are uncommon (1). Whilst segmental fractures have been reported in the literature, concurrent lateral and medial injuries are very rare. These injuries are, therefore, susceptible to being missed, due to failure to look for a second injury after the initial diagnosis, and difficult $\mathrm{X}$-ray interpretation around the area of the medial clavicle. The nature of segmental fractures can pose a difficult management problem for numerous reasons, and initial operative fixation is usually indicated. Early diagnosis is therefore imperative, and as such, clinical examination is essential even if an obvious mid or lateral shaft fracture is seen on X-ray. This unusual case of combined lateral and medial fractures was initially missed and the presentation and management is discussed.
\end{abstract}

\section{Case Report}

A 54-year-old male was assaulted whilst out walking on holiday. $\mathrm{He}$ was uncertain as to the mechanism of injury and chain of events, but recalled being knocked to the ground. A lateral clavicle fracture was diagnosed. $\mathrm{He}$

Dr R Heywood SHO Email:

rebecca_heywood@yahoo.com

Lt Col J Clasper

RAMC (V)

Department of

Orthopaedics and

Trauma Surgery,

Frimley Park Hospital,

Frimley, Surrey,

GU16 5UJ was reassured, given a broad arm sling and referred to the nearest fracture clinic.

On presentation four days after the injury he complained of pain in the collarbone and shoulder. On examination there was obvious deformity and swelling at the lateral end of the clavicle, and tenderness over the medial and lateral ends. There was no tenderness of the shoulder or scapula but reduced range of movement at the shoulder secondary to pain in the clavicle on shoulder movement. There was no neurovascular deficit and no other significant injuries.

Radiographs brought from the time of injury showed the anteriorly displaced right lateral clavicle fracture. In addition a posteriorly displaced fracture of the medial end was clearly seen (Figure 1).

Open reduction and internal fixation was performed the next day (Figure 2). Following this mobilisation and physiotherapy was started. Bony consolidation was evident radiographically twelve weeks after operation.

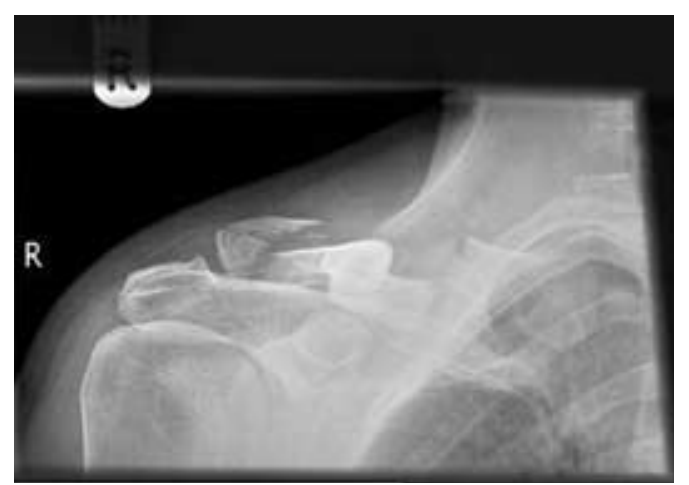

Fig 1. Segmental clavicle fracture.

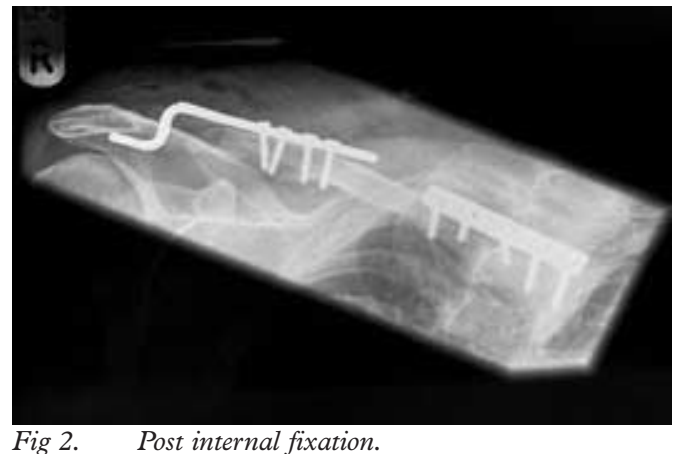

\section{Discussion}

Among clavicle fractures, $69 \%$ occur in the midshaft, $28 \%$ at the lateral end, and the remaining $3 \%$ at the medial end (1). Segmental fractures have been reported but usually combine midshaft and lateral clavicle injuries or clavicle fracture with proximal clavicular physis injury in children $(2,3)$.

The overlying structures in the chest can make it difficult to assess the medial clavicle well on X-ray. The rarity of double clavicle injuries and indeed of solitary medial ones would also preclude one from identifying a second fracture. However, in this case the Xray findings were obvious, and the injury should have been diagnosed by a thorough examination of the patient and X-ray 
evaluation.

Clavicle fractures are most commonly the result of simple falls resulting in direct force on to the point of the shoulder (1). Other cases of double clavicle fractures have been thought to be caused by separate blows and this would seem to be the case here $(2,3)$. It is possible that the medial injury was sustained by a fall to the ground, with the lateral fracture as a result of a direct blow, for example, kick to the lateral aspect of the shoulder, or that both fractures resulted from kicks or direct forces in succession from a blunt object. A history of more than one force applied to the clavicle must prompt one to seek more than one injury.

Although the majority of clavicle fractures can be managed non-operatively in a broad arm sling and are considered of low consequence, operative management is indicated for more complex injuries such as open fractures, neurovascular compromise or floating shoulder, and complications such as symptomatic non-union (4). In general, segmental fractures are also fixed due to the risk of non-union. The clavicle forms one of the struts of the shoulder girdle and is essential for the support and function of the upper limb on the axial skeleton. Non-union of a clavicle fracture can, therefore, have considerable consequences in terms of functional deficit and pain, and also have complications at the medial site due to the large vessels lying posterior (4). Primary operative treatment has also previously been recommended for all displaced lateral end fractures due to the high incidence of nonunion (1). One of the most significant predisposing factors to non-union is the initial degree of displacement of the fracture fragments, irrespective of the mechanism of injury $(1,4)$. Both fractures in this case were displaced.

Segmental long bone fracture almost invariably results in rapid union at one fracture site and delayed or non-union at the other, though most of the literature concerns segmental tibial fractures. These are renowned for their high risk of complications and non-operative treatment is considered unacceptable. The same principles can be applied to the clavicle. It is proposed that the high non-union rate at one fracture site occurs due to the subcutaneous position of the bone and the associated direct injury to the surrounding soft tissues. The intermediate segment is problematic due to the uncertain blood supply and lack of control over its position due to the multiple forces acting on it from attached muscles. Thus segmental clavicle fractures must be fixed, and therefore diagnosed, early.

Although in this case the diagnosis was made only four days post injury and the patient, therefore, suffered no complications, he could have easily done so due to the high risk of non-union resulting from the segmental nature of the injury, the site and displacement of the fractures. The consequences of non-union in a relatively young patient, in whom functional deficit would be problematic, prompted primary surgical stabilisation. This case must alert us to evaluate the patient by clinical examination whatever the initial X-ray findings, and to always consider a segmental injury when fracture of the clavicle is diagnosed, as they should be referred for consideration of operative fixation.

\section{References}

1. Robinson CM. Fractures of the clavicle in the adult. f Bone foint Surgery (Br) 1998;80:476-84.

2. Hagino $T$, Ono $T$, Hamada $Y$. Unusual double clavicle fracture complicated by ipsilateral scapular neck fracture. F Orthop Sci 2002;7:417-9.

3. O'Neill P, Cosgarea A, McFarland E. Unusual double clavicle fracture in a lacrosse player. $\operatorname{Clin} \mathcal{F}$ Sport Med 2000;10:69-71.

4. Jupiter J, Leffert R. Non-union of the clavicle. F Bone foint Surgery (Am) 1987;69-A:753-60. 